\title{
Changes in the Thickness of Trunk Stabilizer Muscles According to Increased Lifting Loads in Stoop Lifting
}

\author{
Hoe-Song Yang, PT, MSc'1), Oh-Yun Kwon, PT, PhD²), Yeon-Seop Lee, PT, PhD ${ }^{1)}$ \\ 1) Department of Physical Therapy, Cheongam College \\ 2) Department of Physical Therapy, College of Health Science, Yonsei University: 234 Maeji-ri, \\ Heungup-myon, Wonju, Kangwon-do, Republic of Korea. TEL: +82 33-760-2971, FAX: +82 33-760- \\ 2496,E-mail:kwonoy@yonsei.ac.kr
}

\begin{abstract}
Purpose] The aim of this study was to identify how the lumbar stabilizer muscles respond to increased lifting loads. Twenty-four healthy subjects (10 males, 14 females) participated in this study. [Subjects and Methods] The thicknesses of the internal oblique (IO), transverse abdominis (TrA), and lumbar multifidus (LM) muscle were measured by ultrasonography during lifting of loads $10 \%, 20 \%$, and $30 \%$ of body weight. The data was analyzed measured by one-way repeated measures analysis of variance (ANOVA). [Results] There were statistically significant increases in thicknesses of the TrA and LM muscles when lifting a load of $20 \%$ of subject's body weight. The thickness of IO was not significantly different at different loads. [Conclusion] The findings of this study suggest that $\operatorname{Tr} \mathrm{A}$ and LM play important roles as lumbar spine stabilizers during lifting activities of less than $20 \%$ of body weight. Further study is needed to find the mechanisms of lumbar stability during stoop lifting of loads greater than $20 \%$ of body weight.

Key words: Lumbar stabilizers, Lifting load, Ultrasonography
\end{abstract}

(This article was submitted Sep. 5, 2012, and was accepted Oct. 5, 2012)

\section{INTRODUCTION}

Repeated lifting motions at work and in daily life are a major cause of musculoskeletal disorders such as low back pain ${ }^{1)}$. Lifting motions involve increases in intra-abdominal pressure achieved through simultaneous contraction of the back and abdominal muscles $\left.{ }^{2}, 3\right)$. The abdominal muscles serve important roles in lumbar stabilization when loads are imposed on the trunk ${ }^{4}$.

The lumbar stabilizer muscles are divided into global and local muscles based on their dynamic roles. The global muscles include the erector spinae, the rectus abdominis, the internal oblique (IO), and external oblique (EO) abdominal muscles. They induce movement or directly deliver loads to the pelvis and the thoracic cage, and act when large external loads are imposed on the spine ${ }^{5)}$. The local muscles include the LM (lumbar multifidus) and the transversus abdominis (TrA). They act to maintain posture and adjust movements between the vertebrae when small loads are imposed ${ }^{6}$. Effective interactions of these muscles produce effective and safe lifting movements.

The general guidelines for safe lifting state that squat lifting postures are appropriate for reducing loads on the non-contractile tissues in the lower lumbar spine ${ }^{7,8)}$. However, many workers prefer stoop lifting because these postures consume less energy and facilitate balance during lifting ${ }^{9)}$. However, stoop lifting at work and in daily living activities raise the risk of spinal injury.
Studies of biomechanical changes in the trunk muscles related to lifting motions have been performed, including those of muscle fatigue in static and dynamic conditions ${ }^{10)}$, symmetrical or asymmetrical working postures used in lifting ${ }^{11)}$, global muscle activities related to the degree of load $^{12)}$, the angles of the trunk during lifting ${ }^{13)}$, and lifting speeds ${ }^{14)}$. However, these studies focused on the global muscles and working postures related to lifting. Studies of the actions of the lumbar stabilizer muscles during lifting motions in relation to the degree of load are insufficient. Furthermore, unlike individual muscles in the extremities, the force necessary for the movements of the lumbar stabilizer muscles cannot be directly measured. Also, surface electromyography cannot measure the muscle activities of deep muscles. Therefore, non-invasive ultrasonography is a useful tool for measuring the changes in thicknesses of deep muscles ${ }^{15,16)}$.

The present study examined the changes in the muscle thicknesses of the trunk stabilizer muscles, IO, TrA, and LM, at different loads in stoop lifting postures through measurement using ultrasonography.

\section{SUBJECTS AND METHODS}

\section{Subjects}

The subjects of the present study were 24 normal adults (10 males, 14 females). All the subjects agreed to participate in the study after hearing an explanation about the experi- 
Table 1. General characteristics of subjects $(\mathrm{N}=24)$

\begin{tabular}{cc}
\hline Characteristics & Mean \pm SD \\
\hline Age $(\mathrm{yrs})$ & $20.7 \pm 0.9$ \\
Height $(\mathrm{cm})$ & $167.4 \pm 8.3$ \\
Mass $(\mathrm{kg})$ & $60.9 \pm 8.0$ \\
$\mathrm{BMI}^{\mathrm{a}}\left(\mathrm{kg} / \mathrm{m}^{2}\right)$ & $21.6 \pm 2.0$ \\
\hline
\end{tabular}

${ }^{\mathrm{a} B o d y}$ Mass Index

mental method. They were selected from those who had no experience of low back pain within the previous 6 months and had no orthopedic or neurological disease of the spine or lower extremities. The general characteristics of the study subjects are presented in Table 1.

\section{Methods}

Changes in the thicknesses of the trunk stabilizer muscles were measured in lifting motions with loads equal to $10 \%$, $20 \%$ and $30 \%$ of each subject's body weight. The lifting motions were performed using a back strength dynamometer (Takei, Japan). A strain gauge (Noraxon Telemyo 1.06 software, Noraxon Inc., Scottsdale, AZ, U.S.A.) was used to accurately measure and monitor the amount of the load during lifting.

The thickness of the deep lumbar muscles was measured using a SonoAce X4 (Medison Co. Ltd., Seoul, Korea). Measuring points were marked with a ball-point pen to ensure the probe was positioned over same site for each trial. The thickness of the TrA and the IO muscles were measured using a $5-7 \mathrm{MHz}$ linearly arranged probe. The thickness of the LM muscle was measured using a $5-7 \mathrm{MHz}$ curved probe (Fig. 1).

The thickness of each muscle was normalized with the mean value of the muscle thickness measured in the resting position. The thicknesses of TrA and IO in the resting position were measured in the supine position with the knee joint and hip joint maintained at $50^{\circ}$ and $90^{\circ}$, respectively. Thickness of the LM muscle in the resting position was measured in the prone position. Lumbar curvature was minimized by providing abdominal support with a pillow.

To measure the thicknesses of TrA and IO, the probe was placed at the lateral margin $25 \mathrm{~mm}$ anterior to the halfway point between the upper iliac crest and the subcostal angle on the center line of the right axilla. The probe was adjusted so that the lateral abdominal muscles were clear in a direction parallel to the muscle fibers of $\operatorname{TrA}^{17,18)}$. All ultrasonography images were collected at the end of exhalation in order to minimize the respiratory mobilization of the TrA. The thickness was measured after drawing a vertical line at the $25 \mathrm{~mm}$ position in the horizontal plane on the most medial side of $\operatorname{TrA}^{19}$ ).

Before making measurements, the thickness, L4-5 spinous processes were palpated and marked. Then the transducer was placed upright on the centerline of the lumbar spine, and the facet joint was made to appear on the center of the monitor by moving the probe placed longitudinally until the
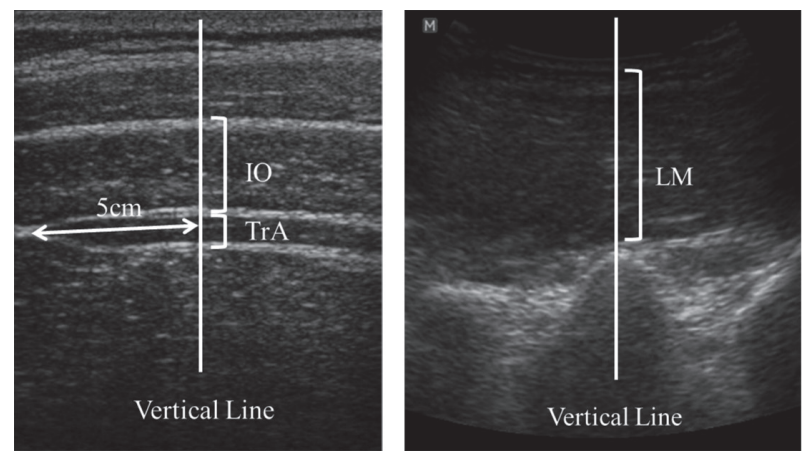

Fig. 1. Ultrasound images of the internal oblique (IO), transversus abdominis (TrA), and lumbar multifidus (LM) muscles

spinal facet joint was clearly seen ${ }^{20)}$.

To measure muscle thickness during stoop lifting, the subject was instructed to bend the trunk to $30^{\circ}$ without bending the knees (stoop lifting), and to remain forward and not raise the shoulders or extend the trunk during the measurement. The principal investigator checked the amount of the load on the monitor connected to the strain gauge. Muscle thickness was measured at the endpoint of exhalation while maintaining the lifting position for 5 seconds. The different loads were given in random orders. The mean value in the three trials was used for data analysis. To minimize muscle fatigue, one-minute's rest was enforced between trials.

Statistical analyses were conducted using PASW 18.0 for Windows. One-way repeated measures ANOVA was used to examine changes in muscle thickness in relation to the size of the load. The paired t-test was used post hoc to identify significant differences. Significance was accepted for values of $\alpha<0.05$.

\section{RESULTS}

The thicknesses of both the TrA and the LM muscles significantly differed among the lifting loads. The thicknesses of both TrA and LM were greatest at $20 \%$ of body weight. The thicknesses of IO did not significantly differ among the lifting loads (Table 2) ${ }^{21)}$.

\section{DISCUSSION}

Among work performed using the hands, lifting activity requires stability of the spine for prevention of injury. Co-activation of the back and abdominal muscles is essential for increasing spinal stiffness. Also, stability needs to increase as the load to be lifted increases ${ }^{22)}$. Granata and Orishimo ${ }^{4}$ stated that the abdominal muscles contribute to the maintenance of normal posture when performing daily activities, and lifting loads safely and effectively. Especially, stoop lifting with $30-60^{\circ}$ trunk flexion posture is often used at work and in daily living activities ${ }^{23}$. Bergmark $^{6}$ ) divided the trunk muscles into the local and global muscle systems. The global muscle (IO) and local muscles (TrA and LM) are 
Table 2. The thicknesses of the lumbar stabilizers at different loads in stoop lifting

\begin{tabular}{ccccc}
\hline Muscles & $10 \%$ & $20 \%$ & $30 \%$ & Post hoc \\
\hline IO & $10.1 \pm 2.3^{\mathrm{a}}$ & $10.5 \pm 2.1$ & $10.3 \pm 2.5$ & $/$ \\
TrA & $3.0 \pm 0.6$ & $3.3 \pm 0.4$ & $3.0 \pm 0.5^{*}$ & $\mathrm{a}<\mathrm{b}, \mathrm{b}>\mathrm{c}^{\mathrm{b}}$ \\
LM & $29.2 \pm 4.2$ & $30.2 \pm 4.1$ & $30.0 \pm 4.1^{*}$ & $\mathrm{a}<\mathrm{b}$ \\
\hline
\end{tabular}

(unit: $\mathrm{mm}$ ) ${ }^{\mathrm{a}}$ Mean \pm standard deviation, ${ }^{\mathrm{b}} \mathrm{a}: 10 \%$, b: $20 \%$, c: $30 \%,{ }^{*} \mathrm{p}<0.05$. IO: internal oblique, TrA: transversus abdominis, LM: lumbar multifidus

involved in the stability of the trunk. Therefore, the present study investigated whether the thicknesses of the TrA, IO, and LM muscles increase when lifting loads of $10 \%, 20 \%$, and $30 \%$ of the body weight in a stoop lifting posture.

The result of this study show that the thickness of the TrA increased more significantly when the load was $20 \%$ of the subject's body weight than when the load was $10 \%$ or $30 \%$. Hide et al. ${ }^{15)}$ examined the thicknesses of the TrA and IO using ultrasonography during rest, while lifting with a load of $25 \%$ of body weight during a simulated unilateral weightbearing task against a footplate in the supine position. They reported that the thicknesses of TrA and IO significantly increased under the load of $25 \%$ of body weight compared to at rest, and that the thickness of the TrA increased more than IO. Granata and Orishimo ${ }^{4}$ conducted a biomechanical model study of lifting weights $(4.5$ and $9.0 \mathrm{~kg}$ ) and heights $(0,20,40,60$, and $80 \mathrm{~cm})$ in static lifting postures with 20 normal adults. They reported that as the weight and height increased, the activity of the abdominal and back muscles increased. They stated that abdominal muscles acted to maintain the stability of the trunk through co-contraction with the erector spinae muscle, and that if the erector spinae muscle were to contract alone during lifting motions, the spine would become unstable. In our study, the thickness of TrA significantly increased at a load of $20 \%$ body weight compared to the $10 \%$ load. However, the thickness of TrA at the load of $30 \%$ body weight was significantly decreased compared to the $20 \%$ load. This was an interesting result.

The muscle thickness of LM showed a more significant increase at the load of $20 \%$ of body weight than the load of $10 \%$ body weight. However, there was no significant difference in the thickness of LM between loads of $20 \%$ and $30 \%$ body weight. When loads exceeding $20 \%$ of body weight were lifted, the muscle thickness of a TrA, and LM decreased. With regard to changes in muscles related to lifting motions, Olson ${ }^{12)}$ examined the activities of the muscles around the lumbar spine, such as the rectus abdominis, and the EO muscles, in isometric lumbar extension performed by 13 normal adults with forces equal to $50 \%$ and $70 \%$ of MVIC (maximum voluntary isometric contraction) in a posture with trunk flexion of $30^{\circ}$. According to their results, the muscles around the lumbar spine showed no differences between $50 \%$ and $70 \%$, while the abdominal muscles showed more significant increases in muscle activity at $50 \%$ than at $70 \%$. Cholewicki et al. ${ }^{3)}$ reported that when loads equal to $0 \%, 20 \%$, and $40 \%$ of body weight were imposed on the waists of 12 subjects, the stability index increased as the weight increased. The TrA and LM muscles act as local muscles, and local muscles contribute to the maintenance of mechanical stiffness of the spine, controlling intersegmental motion rather than producing large torque. The TrA and LM muscles can maintain lumbar stability at loads below $20 \%$ loads of body weight, but other global muscles seem to be activated to maintain lumbar stability at loads above $20 \%$ of body weight. This would be a possible reason why the thicknesses of TrA and LM significantly decreased under the 30\% load condition. Another possible reason is the validity of the measurement of muscle thickness using ultrasonography. Hodges et al. ${ }^{24)}$ asserted that ultrasound imaging can be used to detect low levels of muscle activity, but that it cannot discriminate between moderate and strong contraction. We did not measure the muscle activities of TrA and LM directly, and a load of $30 \%$ load of body weight may induce strong contractions in TrA and LM. This is another possible reason why the thicknesses of $\operatorname{TrA}$ and LM did not increase under the load of $30 \%$ of body weight. IO did not show any statistically significant differences among the different loads. Hodges et al. ${ }^{24)}$ reported that ultrasound measures reliably corresponded with changes in EMG of 22\% MVC (maximum voluntary contraction) in IO. Although the thickness of IO increased at $20 \%$, there was no significant difference from the road of $10 \%$ of body weight. Further study is needed to confirm whether EMG activity also shows no significant change among the different lifting loads.

The present study had some limitations. The thicknesses of $\operatorname{TrA}$, LM, and IO were measured in static position. Therefore, the results of this study cannot be generalized to dynamic lifting techniques. We did not measure other global muscles, or lumbar stabilizers such as pelvic floor, diaphragm and lower extremity muscles. Further studies are needed to find the interactions between local and global muscles during stoop lifting.

In conclusion, the TrA and the LM muscles contribute to lumbar stabilization at loads of $20 \%$ of body weight in stoop lifting motions. Further studies are needed to find the mechanisms of lumbar stability and the coordination of local and global muscles during stoop lifting of loads greater than $20 \%$ of body weight.

\section{REFERENCES}

1) Straker L: Evidence to support using squat, semi-squat and stoop techniques to lift low-lying objects. Int J Ind Ergon, 2003, 31: 149-160. [CrossRef]

2) Bunce SM, Moore AP, Hough AD: M-mode ultrasound: a reliable measure of transversus abdominis thickness? Clin Biomech (Bristol, Avon), 2002, 17: 315-317. [Medline] [CrossRef]

3) Cholewicki J, Simons AP, Radebold A: Effects of external trunk loads on lumbar spine stability. J Biomech, 2000, 33: 1377-1385. [Medline] [CrossRef]

4) Granata KP, Orishimo KF: Response of trunk muscle co-activation to 
changes in spinal stability. J Biomech, 2001, 34: 1117-1123. [Medline] [CrossRef]

5) Marras WS, Mirka GA: Muscle activities during asymmetric trunk angular accelerations. J Orthop Res, 1990, 8: 824-832. [Medline] [CrossRef]

6) Bergmark A: Stability of the lumbar spine. A study in mechanical engineering. Acta Orthop Scand Suppl, 1989, 230: 1-54. [Medline]

7) Larivière C, Gagnon D, Loisel P: A biomechanical comparison of lifting techniques between subjects with and without chronic low back pain during freestyle lifting and lowering tasks. Clin Biomech (Bristol, Avon), 2002, 17: 89-98. [Medline] [CrossRef]

8) Faber GS, Kingma I, Bruijn SM, et al: Optimal inertial sensor location for ambulatory measurement of trunk inclination. J Biomech, 2009, 42: 2406-2409. [Medline] [CrossRef]

9) Hagen KB, Hallen J, Harms-Ringdahl K: Physiological and subjective responses to maximal repetitive lifting employing stoop and squat technique. Eur J Appl Physiol Occup Physiol, 1993, 67: 291-297. [Medline] [CrossRef]

10) Bonato $P$, Gerold R, Ebenbichler MD, et al.: Muscle fatigue and fatiguerelated mechanical changes during a cyclic lifting task. Spine, 2003, 28 : 1810-1820. [Medline] [CrossRef]

11) Chow DH, Cheng IY, Holmes AD, et al.: Muscular and center of pressure response to sudden release of load in symmetric and asymmetric stoop lifting tasks. Appl Ergon, 2005, 36: 13-24. [Medline] [CrossRef]

12) Olson MW: Trunk muscle activation during sub-maximal extension efforts. Man Ther, 2010, 15: 105-110. [Medline] [CrossRef]

13) Tan JC, Parnianpour M, Nordin $M$, et al.: Isometric maximal and submaximal trunk extension at different flexed positions in standing: triaxial torque output and EMG. Spine, 1993, 18: 2480-2490. [Medline] [CrossRef]

14) Yoon J, Shiekhzadeh A, Nordin M: The effect of load weight vs. pace on muscle recruitment during lifting. Appl Ergon, 2012, 43: 1044-1050.
[Medline]

15) Hides JA, Wong I, Wilson SJ, et al.: Assessment of abdominal muscle function during a simulated unilateral weight-bearing task using ultrasound imaging. J Orthop Sports Phys Ther, 2007, 37: 467-471. [Medline]

16) Koppenhaver SL, Hebert JJ, Fritz JM, et al.: Reliability of rehabilitative ultrasound imaging of the transversus abdominis and lumbar multifidus muscles. Arch Phys Med Rehabil, 2009, 90: 87-94. [Medline] [CrossRef]

17) Critchley DJ, Coutts FJ: Abdominal muscles function in chronic low back pain. Physiotherapy, 2002, 68: 322-331. [CrossRef]

18) McMeeken JM, Beith ID, Newham DJ, et al.: The relationship between EMG and change in thickness of transversus abdominis. Clin Biomech (Bristol, Avon), 2004, 19: 337-342. [Medline] [CrossRef]

19) Ferreira PH, Ferreira ML, Hodges PW: Changes in recruitment of the abdominal muscles in people with low back pain: ultrasound measurement of muscle activity. Spine, 2004, 29: 2560-2566. [Medline] [CrossRef]

20) Hides J, Gilmore C, Stanton W, et al.: Multifidus size and symmetry among chronic LBP and healthy asymptomatic subjects. Man Ther, 2008, 13: 43-49. [Medline] [CrossRef]

21) Sánchez-Zuriaga D, Vera-Garcia FJ, Moreside JM, et al.: Trunk muscle activation patterns and spine kinematics when using an oscillating blade: influence of different postures and blade orientations. Arch Phys Med Rehabil, 2009, 90: 1055-1060. [Medline] [CrossRef]

22) Cholewicki J, McGill SM: Mechanical stability of the in vivo lumbar spine implications for injury and chronic low back pain. Clin Biomech (Bristol, Avon), 1996, 11: 1-15. [Medline] [CrossRef]

23) Nordin M, Ortengren R, Andersson GB: Measurements of trunk movement during work. Spine, 1984, 9: 465-469. [Medline] [CrossRef]

24) Hodges PW, Pengel LH, Herbert RD, et al.: Measurement of muscle contraction with ultrasound imaging. Muscle Nerve, 2003, 27: 682-692. [Medline] [CrossRef] 